CERN-PPE/92-28

12 February 1992

\title{
STUDY OF CHARM PHOTOPRODUCTION MECHANISMS
}

\author{
M.P. Alvarez ${ }^{2)}$, R. Barate ${ }^{3, b)}$, D. Bloch ${ }^{9)}$, P. Bonamy ${ }^{7)}$, P. Borgeaud ${ }^{7)}$, M. Burchell ${ }^{4)}$,
} H. Burmeister ${ }^{3)}$, J.M. Brunet ${ }^{6)}$, F. Calvino ${ }^{2, a)}$, M. Cattaneo ${ }^{4)}$, J.M. Crespo ${ }^{2)}$, B. d'Almagne ${ }^{5)}$, M. David ${ }^{7)}$, L. di Ciaccio $^{3, c)}$, J. Dixon ${ }^{4)}$, P. Druet ${ }^{5)}$, A. Duane ${ }^{4)}$, J.P. Engel ${ }^{9)}$, A. Ferrer ${ }^{3, d)}$, T.A. Filippas ${ }^{1)}$, E. Fokitis ${ }^{1)}$, R.W. Forty ${ }^{4)}$, P. Foucault ${ }^{9)}$, E.N. Gazis ${ }^{1)}$, J.P. Gerber ${ }^{9)}$, Y. Giomataris ${ }^{3)}$, T. Hofmokl ${ }^{10)}$, E.C. Katsoufis ${ }^{1}$, M. Koratzinos ${ }^{3,4,5)}$, C. Krafft ${ }^{5)}$, B. Lefievre ${ }^{6)}$, Y. Lemoigne ${ }^{7)}$, A. Lopez ${ }^{3,5, *)}$, W.K. Lui ${ }^{8)}$, C. Magneville ${ }^{7)}$, A. Maltezos ${ }^{1)}$, J.G. McEwen ${ }^{8)}$, Th. Papadopoulou1 ${ }^{1)}$, B. Pattison ${ }^{3}$, D. Poutot ${ }^{6)}$, M. Primout ${ }^{7)}$, H. Rahmani ${ }^{1)}$, P. Roudeau ${ }^{5)}$, C. Seez ${ }^{4)}$, J. Six ${ }^{5)}$, R. Strub ${ }^{9)}$, D. Treille ${ }^{3)}$, P. Triscos ${ }^{6)}$, G. Tristram ${ }^{6)}$, G. Villet ${ }^{7)}$, A. Volte ${ }^{6)}$, M. Wayne ${ }^{5)}$, D.M. Websdale ${ }^{4)}$, G. Wormser ${ }^{5)}$ and Y. Zolnierowski ${ }^{3)}$

\section{(The NA14/2 Collaboration)}

\footnotetext{
Abstract

(Submitted to Zeitschrift für Physik C)

1) National Technical University, Athens, Greece.

2) Universidad Autónoma de Barcelona, Bellaterra, Spain.

3) CERN, Geneva, Switzerland.

4) Blackett Lab., Imperial College, London, UK.

5) LAL, IN2P3-CNRS and Univ. Paris-Sud, Orsay, France.

6) Collège de France, Paris, France.

7) DPhPE, CEN-Saclay, Gif-sur Yvette, France.

8) Univ of Southampton, UK.

9) CRN, IN2P3-CNRS and Univ. L. Pasteur, Strasburg, France.

10) University of Warsaw, Poland.

a) Univ. Politecnica de Catalunya, ETSEIB-DEN, Barcelona, Spain.

b) Univ. J. Fourier and ISN, Grenoble, France.

c) Univ. di Roma II, 'Tor Vergata', Rome, Italy.

d) Univ. de Valencia, IFIC, Spain.

*) On leave from Fac. de Ciencas Fisicas, Univ. Complutense, Madrid, Spain.
}

We present results on charm photoproduction in the energy interval 40 to $160 \mathrm{GeV}$, obtained from high-statistics charm samples at the NA14/2 experiment at CERN. We have measured the charm cross-section, $x_{\mathrm{F}}$ and $p_{\mathrm{T}}^{2}$ distributions, and various production ratios and charge asymmetries. The total non-diffractive open charm cross-section per nucleon is measured to be $\sigma_{(\gamma \mathrm{N} \rightarrow c \bar{c} X)}=465 \pm 53 \pm 84 \mathrm{nb}$ at $\left\langle E_{\gamma}\right\rangle=$ $100 \mathrm{GeV}$. The hadronization scheme is discussed, as well as the comparison with theory and phenomenological models. The comparison of the $p_{\mathrm{T}}^{2}$ distribution with first-order QCD calculations yields an estimate for the charm quark mass of $m_{\mathrm{c}}=1.58 \pm 0.07 \mathrm{GeV} / c^{2}$. 

Introduction

The photoproduction of charm has several attractive features: it can be described in the framework of QCD owing to the fact that the charm-quark mass is large; perturbative expansion is therefore valid even for low- $p_{\mathrm{T}}$ values, in contrast to the photoproduction of light flavours. To lowest order in $\alpha_{\mathrm{S}}$ only one process contributes, that of gamma-gluon $(\gamma \mathrm{g})$ fusion. Higher-order corrections are expected to be small and the second-order contribution has been calculated [1]. To this order, only the gluon structure function is involved. The theoretical uncertainties are much smaller than in hadroproduction, where higher order corrections are larger and additional structure functions are involved. Furthermore, the charm fraction of the hadronic cross-section is much higher in photoproduction.

The hadronization scheme is also simple. In the framework of the Dual Parton Model (DPM) [2] it is expected to be dominated by a single topological graph (Fig. 1).

Our photoproduction experiment, NA14/2, has collected data using a wideacceptance spectrometer with good electromagnetic calorimeter coverage. Particle identification is provided by two Cherenkov counters and the momentum resolution for a track traversing both magnets is $\Delta p / p^{2} \approx 4 \times 10^{-4}(p$ in $\mathrm{GeV} / c)$. The vertex detector incorporates a silicon active target of 32 planes, each plane segmented into 2 mm wide strips with analogue readout. These are followed downstream by a high-resolution silicon-microstrip detector comprising 10 planes with a $50 \mu \mathrm{m}$ pitch. The microstrip detector was developed by NA14/2 in collaboration with industry [3] and has been successfully used by other photoproduction experiments [4]. The trigger selected hadronic interactions of photons in the silicon active target. Details concerning the detector can be found in Ref. [5].

A study of production mechanisms requires the measurement of the energy of individual photons. The photon beam was created by electron bremsstrahlung, and the electron energies were measured both before and after radiation. The effect of multiple bremsstrahlung is taken into account using a Monte Carlo simulation. The photons range in energy from 40 to $160 \mathrm{GeV}$ with $\left\langle E_{\gamma}\right\rangle=95 \mathrm{GeV}$.

A total of 17 million events has been recorded. Various filtering schemes have been used to extract interesting events before processing them through the complete reconstruction program. In addition, for the photoproduction analysis, 4.3 million events were directly processed through the complete reconstruction program (at the CERN 3081/E 'emulator farm'). These events are used for cross-section measurements to ensure a sample free from filter inefficiencies and biases.

\section{The hadronization scheme}

According to the DPM, the final-state hadronization is dominated by a single topological graph. The hadronization occurs along two 'strings': a mesonic string stretched between the anticharm quark and a target spectator quark, and a baryonic string stretched between the charm quark and the remaining target spectator diquark (Fig. 1). This process is simulated using Monte Carlo techniques.

Our Monte Carlo program uses the first-order QCD calculation for the photoproduction of charm, via the $\gamma \mathrm{g}$ fusion mechanism. In the perturbative QCD calculation of charm photoproduction, the charm quark and antiquark are produced in a similar way; the source of any particle-antiparticle asymmetry must therefore be attributed to hadronization. 
The gluon structure function $x G(x) \sim(1-x)^{5}$ is assumed, no $\mathrm{Q}^{2}$ evolution is introduced, and the mass of the charm quark is taken as $1.5 \mathrm{GeV}$. The structure functions of the nucleon fragments are taken from low- $p_{\mathrm{T}}$ physics; for the quark we assume it to be [2]:

$$
f(x) \sim \frac{1}{\sqrt{ } x}(1-x)^{3 / 2}
$$

giving an $x^{-1 / 2}$ behaviour for the quark and an $x^{3 / 2}$ behaviour for the diquark at low $x$. Hence the mass of the string containing the diquark is higher than the mass of the string containing the quark, as shown in Fig. 2. Owing to phase-space limitations this inequality in the string masses can lead to particle-antiparticle asymmetry for the charmed particles produced during the hadronization process.

Hadrons are created along the two independent strings using the Lund scheme [6] in which quark-antiquark and diquark-antidiquark pairs are created along the length of the string until the available energy has been exhausted.

We are assuming implicitly that, in our energy range, the contribution of diffractive photoproduction of charmed particles is small. Theoretical arguments [1] indicate that the diffractive component is a few per cent of the total charm cross-section. To check this point we have studied events with a small energy deposition in the active target at the main vertex position and have obtained an upper limit for the coherent (diffractive) component of the charm cross-section. This limit is $3 \%$ at the $90 \%$ confidence level [7]: moreover, an a posteriori indication that this assumption is correct is the shape of the measured $x_{\mathrm{F}}$ distribution of $\mathrm{D}$ mesons.

The results of the Monte Carlo simulation are presented in Fig. 3, where the expected production rates for the various charmed particles are given as a function of the incoming photon energy.

$\Lambda_{\mathrm{c}} / \Lambda_{\mathrm{c}}$ asymmetry: a key issue in particle-antiparticle asymmetry is $\Lambda_{\mathrm{c}}$ production, since $\Lambda_{c}$ is the lightest and hence the most abundantly produced charmed baryon. The Lund Monte Carlo used for final-state hadronization generates diquarks and antidiquarks with similar probability. However, the $\Lambda_{c}$ baryon contains a charm quark and is therefore formed in the baryonic string, whilst the $\Lambda_{c}$ contains an anticharm quark, and is formed in the mesonic string. In the mesonic string, $\Lambda_{c}$ production can only occur if the string mass is larger than $3.2 \mathrm{GeV}$ (equal to the mass of the $\Lambda_{c}$ plus the mass of a nucleon), owing to the requirement of baryon-number conservation. The baryonic string, however, when above the $2.3 \mathrm{GeV} \Lambda_{\mathrm{c}}$ threshold, may form a low-mass system with the charm quark and the spectator diquark without stretching further to produce additional diquark-antidiquark pairs. This low-mass system may then decay into a $\Lambda_{c}$ plus additional mesons. This process has a sharp energy dependence, which is treated as follows in the simulation ${ }^{1)}$ : if the baryonic string mass is less than $4 \mathrm{GeV}$, then only $\Lambda_{c}$ baryons are produced; otherwise a DNX system (a D meson, a nucleon, plus additional hadrons) is generated. The value of $4 \mathrm{GeV}$ was chosen to reproduce the $\Lambda_{\mathrm{c}} / \mathrm{D}$ ratio of 0.7 which was measured at $E_{\gamma}=20 \mathrm{GeV}[9]$.

Therefore, at low energies, an excess of $\Lambda_{c}$ over $\Lambda_{c}$ production is expected. This excess is also enhanced by the fact that the mesonic string mass distribution is peaked towards lower values compared to the baryonic string. For high $\Lambda_{c}$ energies, however (above $40 \mathrm{GeV}$ in our case), $\Lambda_{c}$ and $\Lambda_{c}$ production is similar [10].

1) An alternative approach to this problem is given in Ref. [8]. 
D/D asymmetry: the enhancement of D over D production ${ }^{2)}$ results from the $\Lambda_{\mathrm{c}} / \Lambda_{\mathrm{c}}$ asymmetry. As the rate of baryon production is of the order of $10 \%$ of the rate of meson production, the expected excess of $\mathrm{D}$ is only a few per cent.

$\mathrm{D}_{\mathrm{s}}^{+} / \mathrm{D}_{\mathrm{s}}^{-}$asymmetry: the $\mathrm{D}_{\mathrm{s}}^{+} / \mathrm{D}_{\mathrm{s}}^{-}$asymmetry is sensitive to the way charmed hadrons are produced. Owing to phase-space limitations in the mesonic string which suppress $\mathrm{D}_{\mathrm{s}}^{-}$KX production, we predict an excess of $\mathrm{D}_{\mathrm{s}}^{+}$over $\mathrm{D}_{\mathrm{s}}^{-}$. This effect overrides the antiparticle excess expected from the argument for the $\mathrm{D} / \mathrm{D}$ asymmetry.

$\mathrm{D}^{+} / \mathrm{D}^{0}$ ratio: the ratio of $\mathrm{D}^{+}$to $\mathrm{D}^{0}$ production depends on the production rate of prompt $\mathrm{D}^{*}$ mesons. This is because the $\mathrm{D}^{* 0}$ cannot decay to the $\mathrm{D}^{+}$(owing to kinematics), whereas the $\mathrm{D}^{*+}$ decays to $\mathrm{D}^{0}$ or $\mathrm{D}^{+}$with similar probabilities. Taking the branching ratio $\mathrm{BR}\left(\mathrm{D}^{*+} \rightarrow \mathrm{D}^{0} \pi^{+}\right)=0.49 \pm 0.08[11]$ and a ratio of $\left(\mathrm{D}^{0}\right.$ from $\left.\mathrm{D}^{*+}\right) /$ all $\mathrm{D}^{0}=0.32 \pm$ $0.01 \pm 0.03[12]$ gives $\mathrm{D}^{+} / \mathrm{D}^{0}=0.46 \pm 0.06$.

$\left(\mathrm{D}_{\mathrm{s}}^{+}+\mathrm{D}_{\mathrm{s}}^{-}\right) /\left(\mathrm{D}^{+}+\mathrm{D}^{-}\right)$ratio: the production ratio of $\left(\mathrm{D}_{\mathrm{s}}^{+}+\mathrm{D}_{\mathrm{s}}^{-}\right)$to $\left(\mathrm{D}^{+}+\mathrm{D}^{-}\right)$ provides information on strangeness production during hadronization since a charm quark has to combine with a strange quark of the sea to produce a $\mathrm{D}_{\mathrm{s}}$. Thus $\mathrm{D}_{\mathrm{s}}^{+}$production is expected to be suppressed with respect to $\mathrm{D}^{+}$production. If we assume the same probability for strange-quark production as the one used to account for measurements in $\mathrm{e}^{+} \mathrm{e}^{-}$annihilation events, we predict a value of $0.38 \pm 0.15$ for this ratio for incident photon energies greater than $60 \mathrm{GeV}$ [13].

$\Lambda_{c} / \mathrm{D}$ ratio: the production ratio of charmed baryons and antibaryons to $\mathrm{D}$ mesons depends on the rate of diquark generation during the process of hadronization. As threshold effects introduce an asymmetry between $\Lambda_{c}$ and $\Lambda_{c}$, giving an excess of $\Lambda_{c}$ production from direct fusion of a charm quark with a target diquark, we should only compare the rates for $\Lambda_{c}$ photoproduction with the corresponding production in $\mathrm{e}^{+} \mathrm{e}^{-}$annihilation. This comparison will be meaningful for high incident-photon energies where phase-space effects are unimportant. We predict $\Lambda_{c} /\left(\mathrm{D}^{0}+\mathrm{D}^{+}\right)=0.03$ at $E_{\gamma}=90 \mathrm{GeV}$.

\section{Cross-section measurement}

For the measurement of the cross-section, three energy intervals are considered: 40 to $80 \mathrm{GeV}, 80$ to $120 \mathrm{GeV}$, and 120 to $160 \mathrm{GeV}$. The high-statistics modes $\mathrm{D}^{0} \rightarrow \mathrm{K} \pi$ and $\mathrm{D}^{+} \rightarrow \mathrm{K} \pi \pi$ are used for this study ${ }^{3)}$. These are extracted from the directly-processed data, thus avoiding the need for a filter-acceptance correction which would increase the systematic uncertainties. The steps in the analysis to obtain a D signal are described in Ref. [5].

The beam-tagging system provides a measurement of the photon energy in $33 \%$ of the triggers in the data sample. Only tagged events are taken into account for the cross-section measurement.

The choice of the $N_{\sigma}$ cut used (where $N_{\sigma}$ is the ratio of the separation of the primary and secondary vertices scaled by the corresponding error) was made in order to minimize the statistical errors of the final answer. It corresponds to $N_{\sigma}=3$ for the $\mathrm{D}^{0}$ case and

2) Throughout this paper, D stands for the sum of $D^{0}$ and $D^{+}$.

3 ) Thoughout this paper, $\mathrm{D}^{0} \rightarrow \mathrm{K} \pi$ is used as an abbreviation for the decay processes $\mathrm{D}^{0} \rightarrow \mathrm{K}^{-} \pi^{+}$and its charge conjugate $\overline{\mathrm{D}}^{0} \rightarrow \mathrm{K}^{+} \pi^{-} ; \mathrm{D}^{+} \rightarrow \mathrm{K} \pi \pi$ is used as the abbreviation for the $\mathrm{D}^{+} \rightarrow \mathrm{K}^{-} \pi^{+} \pi^{+}$and $\mathrm{D}^{-} \rightarrow \mathrm{K}^{+} \pi^{-} \pi^{-}$processes. Also, throughout this paper the symbol for a particle is taken to include the corresponding antiparticle, unless explicitly stated otherwise. 
$N_{\sigma}=4$ for the $\mathrm{D}^{+}$case. The inclusive spectrum can be seen in Fig. 4 . There are about $390 \pm 30 \mathrm{D}^{0}$ and $260 \pm 30 \mathrm{D}^{+}$events above background. The signal region is taken to be 1.830 to 1.875 for $\mathrm{D}^{0}$ and 1.830 to 1.885 for $\mathrm{D}^{+}$. After background subtraction, the events are corrected for the analysis acceptance according to the momentum of the reconstructed D meson. A cut at $15 \mathrm{GeV} / c$ was introduced to avoid large uncertainties in the (large) acceptance-correction at low D momenta. Its effect as a function of the incoming photon energy is taken into account and is of the order of $20 \%$ for the energy range considered. A cut on the $\mathrm{D}$ momentum was preferred to a cut in $x_{\mathrm{F}}$ since it corresponds better to the nature of the correction; the detector and analysis acceptances are more directly related to the charmed-particle momentum and only indirectly to the fraction of the photon momentum carried by it.

The effect of multiple bremsstrahlung is then taken into account by transforming the tagging answer (i.e. the measured difference of electron energies) distribution to the real photon energy distribution. A transformation matrix for this was derived using a Monte Carlo simulation. The correction has the effect of shifting the mean energy for hadronic events from $\left\langle E_{\text {tagging }}\right\rangle=105 \mathrm{GeV}$ to $\left\langle E_{\gamma}\right\rangle=95 \mathrm{GeV}$ [14].

Finally, the data are corrected for trigger acceptance, again obtained from the NA14 Monte Carlo simulation. This simulation gives a trigger charm-enrichment factor of about 2.0 compared to that of hadronic events. The trigger is measured to be $95 \%$ hadronic.

The cross-section is estimated by comparing our charm-data spectrum with the raw-data (hadronic) spectrum corresponding to the same experimental run period. The total hadronic-photoproduction cross-section for the energy range considered is $115 \mu \mathrm{b}$ [15]. Taking into account the A dependence, as discussed below, this corresponds to $2.47 \mathrm{mb}$ per Si nucleus. However, from this value we have to subtract the diffractive component to which our apparatus is insensitive. This diffractive component is mainly due to diffractive $\rho^{0}$ and $\omega$ production (plus a small contribution from non-resonant $\pi^{+} \pi^{-}$). The cross-section of the reaction $\gamma \mathrm{p} \rightarrow \mathrm{p} \pi^{+} \pi^{-}$(which includes resonant $\rho^{0}$ and non-resonant $\left.\pi^{+} \pi^{-}\right)$is $11 \pm 1 \mu \mathrm{b}$, whereas the cross-section from the diffractive reaction $\gamma \mathrm{p} \rightarrow \mathrm{p} \omega$ is $1.2 \pm 0.3 \mu \mathrm{b}$. Therefore, the total non-diffractive hadronic cross-section is taken to be 103 $\pm 5 \mu \mathrm{b}$. The uncertainty of $5 \%$ represents the systematic uncertainty in our knowledge of both the diffractive part of the cross-section and the acceptance of our trigger for such diffractive events.

To extract the charm photoproduction cross-section for a free nucleon we have to correct for nuclear effects. This is done assuming that the open charm photoproduction has the same behaviour as $\mathrm{J} / \psi$ production. The A dependence of the total hadronic cross-section has the form $\mathrm{A}^{\propto}$ where $\propto=0.920 \pm 0.002[16]$, whereas the incoherent $\mathrm{J} / \psi$ production has a similar form with $\propto=0.94 \pm 0.02 \pm 0.03$ [17]. The difference in the A dependence has a $7 \%$ effect on the ratio of cross-sections of charm to hadronic events and is taken into account.

Using the $\mathrm{D}^{0}$ sample and taking $\mathrm{BR}\left(\mathrm{D}^{0} \rightarrow \mathrm{K} \pi\right)=(4.20 \pm 0.04 \pm 0.04) \%[18]$ we obtain

$$
\sigma_{\left[\left(\mathrm{D}^{0}+\mathrm{D}^{0}\right) / 2\right]}=271 \pm 36 \pm 50 \mathrm{nb} .
$$

For the $\mathrm{D}^{+}$candidates, taking $\mathrm{BR}\left(\mathrm{D}^{+} \rightarrow \mathrm{K} \pi \pi\right)=(9.1 \pm 1.3 \pm 0.04) \%$ [18] we obtain

$$
\sigma_{\left[\left(\mathrm{D}^{+}+\mathrm{D}^{-}\right) / 2\right]}=92 \pm 20 \pm 18 \mathrm{nb} .
$$


To derive the total non-diffractive charm photoproduction cross-section we need to estimate the contribution to the cross-section of the remaining charmed particles, mainly the $D_{\mathrm{s}}$ and the $\Lambda_{\mathrm{c}}$. For the contribution of the $\mathrm{D}_{\mathrm{s}}$ we use the ratio of $\mathrm{D}_{\mathrm{s}} / \mathrm{D}$ derived from our Monte Carlo (Fig. 3), whereas for the $\Lambda_{\mathrm{c}}$, owing to a discrepancy between predicted and observed ratios discussed in Section 5 , we use the observed $\Lambda_{c} / D$ ratio. Thus we derive the sum of the contributions of the $\mathrm{D}^{0}$ and the $\mathrm{D}^{+}$to the total charm cross-section which is $0.78 \pm 0.07$, and the total open charm cross-section is calculated to be:

$$
\sigma_{(\gamma \mathrm{N} \rightarrow \mathrm{c} \bar{c} X)}=465 \pm 53 \pm 84 \mathrm{nb}
$$

$\left\{\sigma_{(\gamma \mathrm{N} \rightarrow \bar{c} \bar{c} X)}\right.$ does not include the (diffractive) contributions from the $\mathrm{J} / \psi$ and $\left.\psi^{\prime}[19]\right\}$. The first error is statistical and the second systematic. The systematic error includes the variation of the overall signal-to-noise ratio, and together with the uncertainties in the analysis- and trigger-acceptance, the tagging-correction, the ratio of $\mathrm{D}$-meson production to overall charm production and the branching fraction. The energy dependence of the charm cross-section for the three intervals considered can be seen in Fig. 5. This figure compares the values obtained with theoretical predictions for various charm-quark masses taken from Ref. [1]. The value of the charm-quark mass of $1.5 \mathrm{GeV}$ reproduces the data well whereas much lighter values seem to be excluded.

\section{$4 \quad x_{\mathrm{F}}$ and $p_{\mathrm{T}}$ distributions}

To benefit from higher statistics, $x_{\mathrm{F}}$ and $p_{\mathrm{T}}^{2}$ distributions were extracted from the filtered samples of $\mathrm{D}$ mesons rather than from the directly-processed sample.

The $x_{\mathrm{F}}$ distribution obtained from the $\mathrm{D}^{0} \rightarrow \mathrm{K} \pi$ and $\mathrm{D}^{0} \rightarrow \mathrm{K} \pi \pi \pi$ samples can be seen in Fig. 6. It is corrected for efficiencies and acceptances for these two modes and for the effect of multiple photon radiation of the incident electron. The predictions for the $x_{\mathrm{F}}$ distribution are presented for $m_{\mathrm{c}}=1.5 \mathrm{GeV} / c^{2}$ and with different parametrizations of the fragmentation function $z f(z)=(1-z)^{a} \mathrm{e}^{-b m_{\mathrm{T}}^{2} / z}$, where $m_{\mathrm{T}}$ is the transverse mass (Fig. 6a). The data are well described by the standard Lund values $a=1.0$ and $b=0.7$. The effect of using different gluon structure functions is also shown: using the parametrization $x G(x) \sim(1-x)^{n}$ the two curves of Fig. $6 \mathrm{~b}$ are for the values $n=5$ and $n=10$. The $x_{\mathrm{F}}$ distribution is quite insensitive to the charm-quark mass: changing the mass of the charm quark from 1.2 to $1.8 \mathrm{GeV} / c^{2}$ decreases the width of the $x_{\mathrm{F}}$ distribution by $4 \%$.

The shape of the $p_{\mathrm{T}}^{2}$ distribution is insensitive to second-order QCD corrections [1] as well as to the exact form of the gluon structure function. It is therefore a good probe for estimating the charm-quark mass. The raw $p_{\mathrm{T}}^{2}$ distribution obtained from the $\mathrm{D}^{0} \rightarrow \mathrm{K} \pi$, $\mathrm{D}^{+} \rightarrow \mathrm{K} \pi \pi$, and $\mathrm{D}^{0} \rightarrow \mathrm{K} \pi \pi \pi$ samples shows a clear excess at high $p_{\mathrm{T}}^{2}\left(>2 \mathrm{GeV}^{2} / c^{2}\right)$ above the background. This is estimated from the wings on each side of the signal and also from events of the signal region after relaxing the cuts imposed by the vertex detector. The background is well described by $\mathrm{e}^{-6 m_{\mathrm{T}}}$, a standard parametrization of inclusive particle production, suggesting that most of this background is from normal hadronic events. The $p_{\mathrm{T}}^{2}$ distribution, after background subtraction and acceptance corrections, is shown in Fig. 7. The Monte Carlo predictions for $m_{\mathrm{c}}=1.2 \mathrm{GeV} / c^{2}, m_{\mathrm{c}}=1.5 \mathrm{GeV} / c^{2}$, and $m_{\mathrm{c}}=$ $1.8 \mathrm{GeV} / c^{2}$ are also shown.

We estimate the charm-quark mass from the $p_{\mathrm{T}}^{2}$ distribution as follows: we calculate the $\chi^{2}$ of the fit of the theoretical prediction to the experimental distribution for six different charm-quark mass hypotheses $(1.2,1.4,1.5,1.6,1.7$, and $1.8 \mathrm{GeV})$ in the $p_{\mathrm{T}}^{2}$ range 
0 to $8.5(\mathrm{GeV} / \mathrm{c})^{2}$. From the six values of $\chi^{2}$ and quoting only the statistical uncertainty, we estimate a value for the charm-quark mass of

$$
m_{\mathrm{c}}=1.58 \pm 0.07 \mathrm{GeV} / c^{2}
$$

\section{$5 \quad$ Production ratios and charge asymmetries}

Production ratios and charge asymmetries of various charmed particles obtained from events containing reconstructed $\mathrm{D}^{0}, \mathrm{D}^{+}, \mathrm{D}_{\mathrm{s}}$, and $\Lambda_{\mathrm{c}}$ are compared with predictions from the hadronization model.

Defining the asymmetry as $A=(N-N) /(N+N)$ we measure

$$
\begin{gathered}
\mathrm{D} / \mathrm{D}=0.93 \pm 0.07, \quad A_{\mathrm{D}}=-0.03 \pm 0.05 \\
\mathrm{D}_{\mathrm{s}}^{+} / \mathrm{D}_{\mathrm{s}}^{-}=1.6 \pm 0.6, \quad A_{\mathrm{D}_{\mathrm{s}}}=+0.24 \pm 0.17 .
\end{gathered}
$$

Our model predicts asymmetries of opposite sign for non-strange and strange charmed mesons: $A_{\mathrm{D}}=-0.07$ and $A_{\mathrm{D}_{\mathrm{s}}}=+0.12$ (see also Fig. 3 ). The data are compatible with these predictions.

The ratio of $\mathrm{D}_{\mathrm{s}}^{+}$over $\mathrm{D}^{+}$times the branching fraction $\mathrm{BR}\left(\mathrm{D}_{\mathrm{s}}^{+} \rightarrow \phi \pi^{+}\right)$is measured to be $(1.81 \pm 0.63) \%$. Assuming a value of $4 \%$ for $\mathrm{BR}\left(\mathrm{D}_{\mathrm{s}}^{+} \rightarrow \phi \pi^{+}\right)$[20], we obtain a value of $\mathrm{D}_{\mathrm{s}}^{+} / \mathrm{D}^{+}=0.45 \pm 0.16$, whereas the value predicted by our model is $0.38 \pm 0.15$ [13] Given this agreement between the predicted and the estimated value for the $D_{s} / D^{+}$, we can evaluate the contribution of the strange D meson to the total charm cross-section. We obtain $D_{s} /\left(D^{0}+D^{+}\right)=0.14 \pm 0.03$, where the error comes from the uncertainty in the probability of producing a strange quark during hadronization in the LUND scheme.

The $\Lambda_{c} / \Lambda_{c}$ ratio, measured in our experimental acceptance $\left(E_{\Lambda_{c}}>30 \mathrm{GeV}\right)$, is measured to be $0.6 \pm 0.3$. The model predicts 0.7 with the standard LUND fragmentation parameters $(a=1.0, b=0.7)$ and 0.9 with a hard fragmentation function $(a=-0.5$, $b=0.7)$. In this kinematical region we also measure a large $\Lambda_{\mathrm{c}}$ production compared to the LUND prediction. This is illustrated by the production ratios $\left(\Lambda_{c} / D^{0}\right)_{\exp } /\left(\Lambda_{c} / D^{0}\right)_{\text {Lund }}=$ $3.2 \pm 1.0$ and $\left(\Lambda_{\mathrm{c}} / \mathrm{D}^{+}\right)_{\exp } /\left(\Lambda_{\mathrm{c}} / \mathrm{D}^{+}\right)_{\text {Lund }}=3.0 \pm 0.8$ deduced assuming a branching fraction $\mathrm{BR}\left(\Lambda_{\mathrm{c}} \rightarrow \mathrm{pK} \pi\right)=0.05[10]$. The measured $\mathrm{D} / \mathrm{D}$ asymmetry is used to estimate $\Lambda_{\mathrm{c}}$ production over the low-energy range where our apparatus is insensitive. Assuming a branching fraction $\operatorname{BR}\left(\Lambda_{c} \rightarrow \operatorname{pK} \pi\right)=5 \%$, we estimate a ratio of $\left(\Lambda_{c}+\Lambda_{c}\right) /(\mathrm{D}+\mathrm{D})=$ $0.15 \pm 0.06$.

The measurement of $\mathrm{D}^{+} / \mathrm{D}^{0}$ and $\mathrm{D}^{*} / \mathrm{D}$ ratios allows us to extract the fraction $F_{\mathrm{V}}=\mathrm{V} /(\mathrm{P}+\mathrm{V})$ where $\mathrm{V}$ and $\mathrm{P}$ signify vector $(\mathrm{V})$ and pseudoscalar $(\mathrm{P})$ charmed mesons, respectively. Defining $R_{1}=\mathrm{D}^{+} / \mathrm{D}^{0}, R_{2}=\left(\mathrm{D}^{0}\right.$ from $\left.\mathrm{D}^{*+}\right) / \mathrm{D}^{0}$, and $B_{*}=\mathrm{BR}\left(\mathrm{D}^{*+} \rightarrow \mathrm{D}^{0} \pi^{+}\right)$, then $F_{\mathrm{V}}$ is given by the relations:

$$
F_{\mathrm{V}} B_{*}=\left(1-R_{1}\right) /\left(1+R_{1}\right) \quad \text { and } \quad F_{\mathrm{V}} B_{*}=R_{2} /\left(1+R_{2}\right),
$$

assuming an equal production of $\mathrm{D}^{0}$ and $\mathrm{D}^{+}$, and of $\mathrm{D}^{*+}$ and $\mathrm{D}^{* 0}$, respectively, at the parton level.

The $\mathrm{D}^{+} / \mathrm{D}^{0}$ production ratio is measured using the reconstructed $\mathrm{D}^{0} \rightarrow \mathrm{K}^{-} \pi^{+}$and $\mathrm{D}^{+} \rightarrow \mathrm{K}^{-} \pi^{+} \pi^{+}$decays and taking the branching ratio of

$$
\frac{\mathrm{BR}\left(\mathrm{D}^{+} \rightarrow \mathrm{K}^{-} \pi^{+} \pi^{+}\right)}{\mathrm{BR}\left(\mathrm{D}^{0} \rightarrow \mathrm{K}^{-} \pi^{+}\right)}=2.17 \pm 0.37[10] .
$$


We obtain $R_{1}=0.34 \pm 0.09 \pm 0.06$ and we expect $0.46 \pm 0.06$ for our model. This corresponds to a value of $F_{\mathrm{V}} B_{*}=0.49 \pm 0.13$. $R_{2}$ is measured to be $0.26 \pm 0.04$, corresponding to a value of $F_{\mathrm{V}} B_{*}=0.35 \pm 0.07$. These two values are combined to give a weighted average $F_{\mathrm{V}} B_{*}=0.38 \pm 0.05$. Using the value $B_{*}=0.49 \pm 0.08$ [11] we obtain $F_{\mathrm{V}}=0.78 \pm 0.15$, in agreement with what is expected from simple spin counting, i.e. 0.75 , a value which is used by the Lund model.

\section{Conclusions}

We have measured the charm photoproduction cross-section of the $\mathrm{D}^{0}$ and $\mathrm{D}^{+}$ mesons in the energy interval 40-160 GeV:

$$
\sigma_{\left[\left(\mathrm{D}^{0}+\mathrm{D}^{0}\right) / 2\right]}=271 \pm 36 \pm 50 \mathrm{nb}, \quad \sigma_{\left[\left(\mathrm{D}^{+}+\mathrm{D}^{-}\right) / 2\right]}=92 \pm 20 \pm 18 \mathrm{nb} .
$$

The total open charm photoproduction cross-section was estimated to be

$$
\sigma_{(\gamma \mathrm{N} \rightarrow \mathrm{c} \bar{c} X)}=465 \pm 53 \pm 84 \mathrm{nb} .
$$

The $p_{\mathrm{T}}^{2}$ distribution of the $\mathrm{D}^{0}$ and the $\mathrm{D}^{+}$allows us to estimate the mass of the charm quark:

$$
m_{\mathrm{c}}=1.58 \pm 0.07 \mathrm{GeV} / c^{2}
$$

The $x_{\mathrm{F}}$ distribution obtained agrees well with our model, being sensitive to the fragmentation function, but quite insensitive to the gluon structure function. Our model predicts an excess of $\mathrm{D}$ over $\mathrm{D}$ and an excess of $\mathrm{D}_{\mathrm{s}}^{+}$over $\mathrm{D}_{\mathrm{s}}^{-}$, and this prediction agrees with our measurements. The fraction (vector/vector + pseudoscalar) $F_{\mathrm{V}}$ is estimated from the data as $F_{\mathrm{V}}=0.78 \pm 0.15$, which is compatible with the value expected from simple spin counting, i.e. 0.75 .

Our results can be interpreted in the framework of the $\gamma \mathrm{g}$ fusion model. With the exception of the ratio $\Lambda_{\mathrm{c}} / \mathrm{D}$, all the measured quantities are in agreement with our QCDbased simulation, which uses the Dual Parton Model and the Lund model for hadronization. 


\section{REFERENCES}

[1] R.K. Ellis and P. Nason, Nucl. Phys. B312 (1989) 551.

[2] A. Capella and J. Tran Thanh Van, Phys. Lett. B93 (1980) 146.

[3] G. Barber et al., Nucl. Instrum. Methods A253 (1987) 530.

[4] J.R. Raab et al., Phys. Rev. D37 (1988) 2391.

[5] M.P. Alvarez et al. (NA14/2 Collaboration), Z. Phys. C47 (1990) 545.

[6] T. Sjöstrand and M. Bengtsson, Comput. Phys. Commun. 43 (1987) 367.

[7] M. Primout, Thèse d'Etat, CEN-Saclay (1987).

[8] M. Fontanaz, B. Pire and D. Schiff, Z. Phys. C11 (1981) 211.

[9] K. Abe et al., Phys. Rev. D33 (1986) 1.

[10] M.P. Alvarez et al. (NA14/2 Collaboration), Phys. Lett. B246 (1990) 256.

[11] G.P. Yost et al., Particle Data Group, Phys. Lett. B204 (1988) 1.

[12] J.C. Anjos et al., Phys. Rev. Lett. 62 (1989) 5.

[13] M.P. Alvarez et al. (NA14/2 Collaboration), Phys. Lett. B246 (1990) 261.

[14] M. Koratzinos, Ph.D. Thesis, Imperial College, London (1991).

[15] H. Schopper, ed., Total cross sections for reactions of high energy particles (LandoltBörnstein, New Series, Vol. 12b, 1987), p. 345.

[16] D.O. Caldwell et al., Phys. Rev. Lett. 42 (1979) 553.

[17] M.D. Sokoloff et al. (E691 Collaboration), Phys. Rev. Lett. 57 (1986) 3003.

[18] J. Adler et al., Phys. Rev. Lett. 60 (1988) 89.

[19] R. Barate et al., Z. Phys. C33 (1987) 505.

[20] J. Adler et al., Phys. Rev. Lett. 60 (1988) 89. 


\section{Figure captions}

Fig. 1 Photon-gluon fusion and the quark hadronization scheme in the Dual Parton Model.

Fig. 2 String masses in the hadronization scheme of Fig. 1 calculated from the Lund Monte Carlo.

Fig. 3 Predictions of charmed-particle production rates.

Fig. 4 D-meson mass spectrum used for the cross-section measurement.

Fig. 5 Photoproduction cross-section measurements as a function of beam energy, with theoretical second-order predictions for three charm-quark masses, obtained from Ref. [1].

Fig. 6 The $x_{\mathrm{F}}$ distribution of $\mathrm{D}^{0}$ mesons normalized for $x_{\mathrm{F}}>0$. The curves show theoretical predictions for different parameters of the fragmentation function (a), and for different gluon structure functions (b).

Fig. 7 The $p_{\mathrm{T}}^{2}$ distribution of $\mathrm{D}$ mesons. The curves are the expected distributions for three different charm-quark masses and for normal hadronic events. 


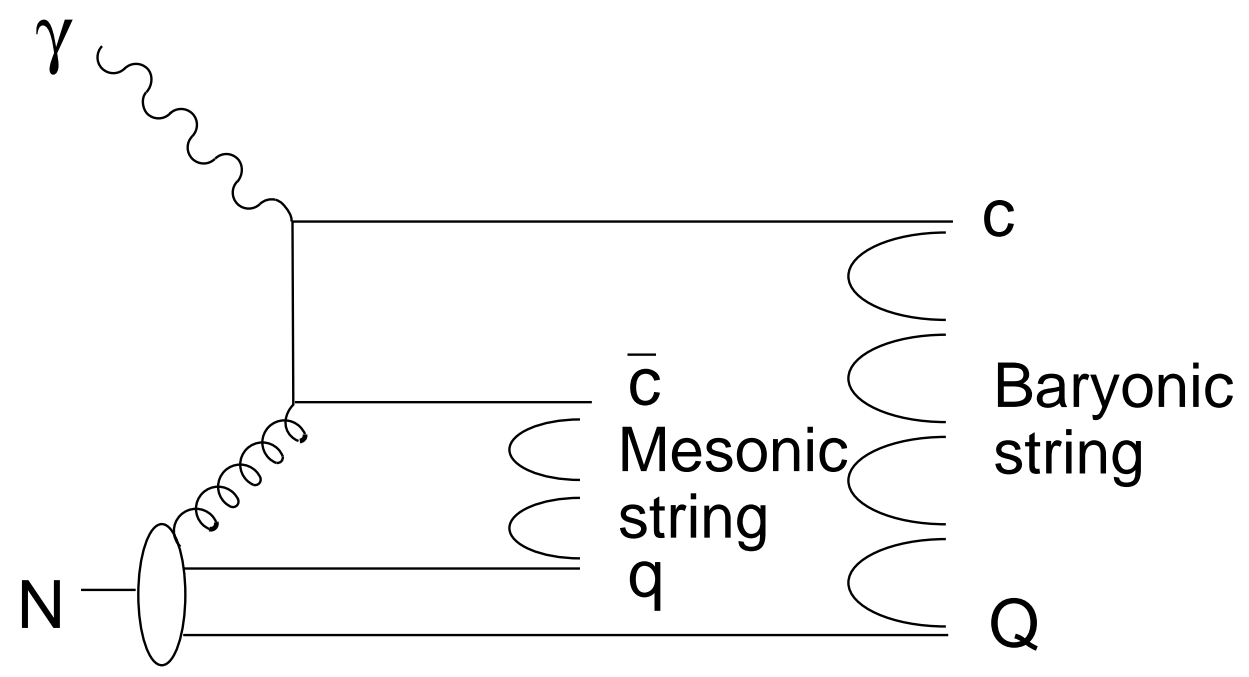

Figure 1

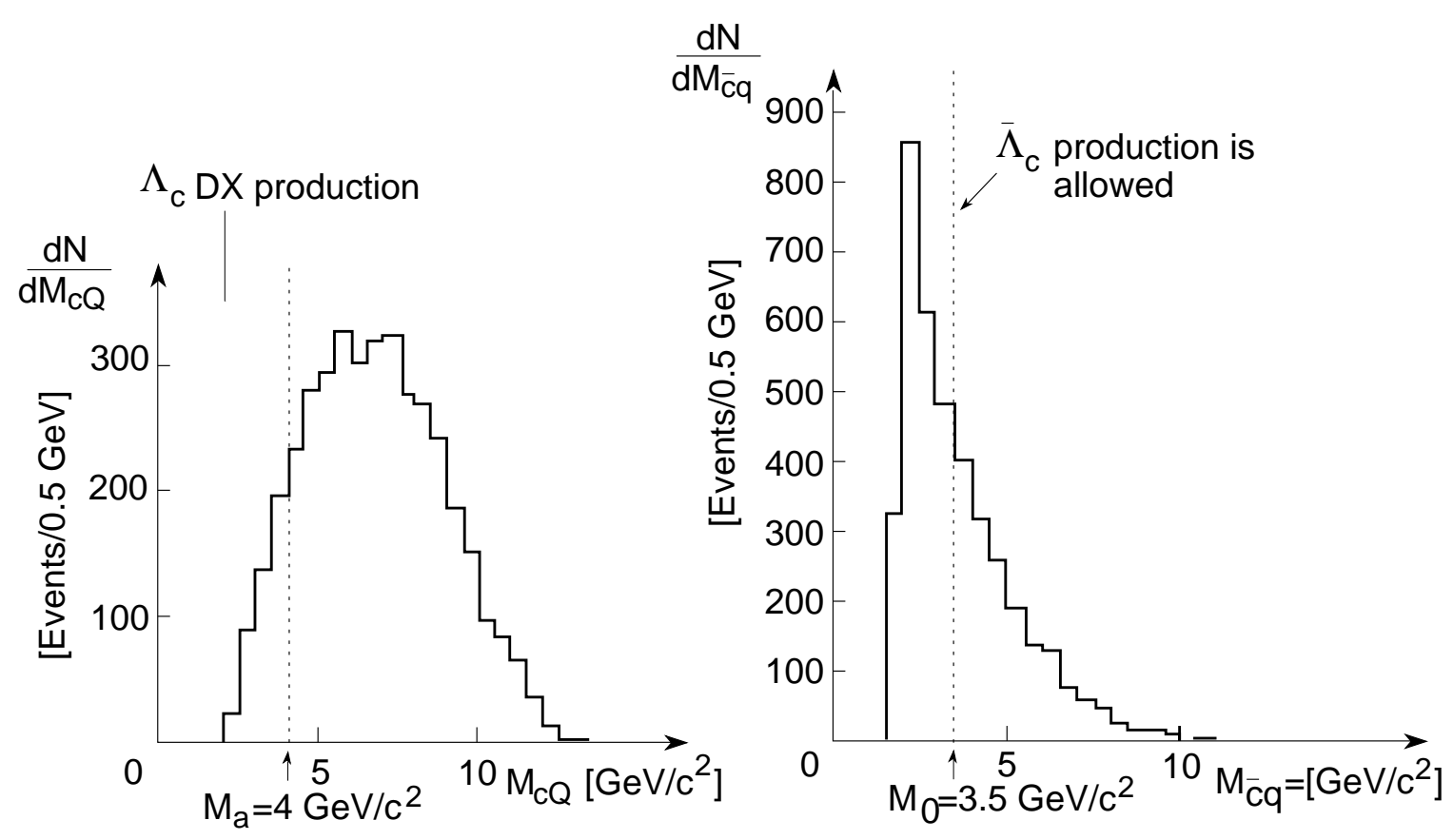

Figure 2 


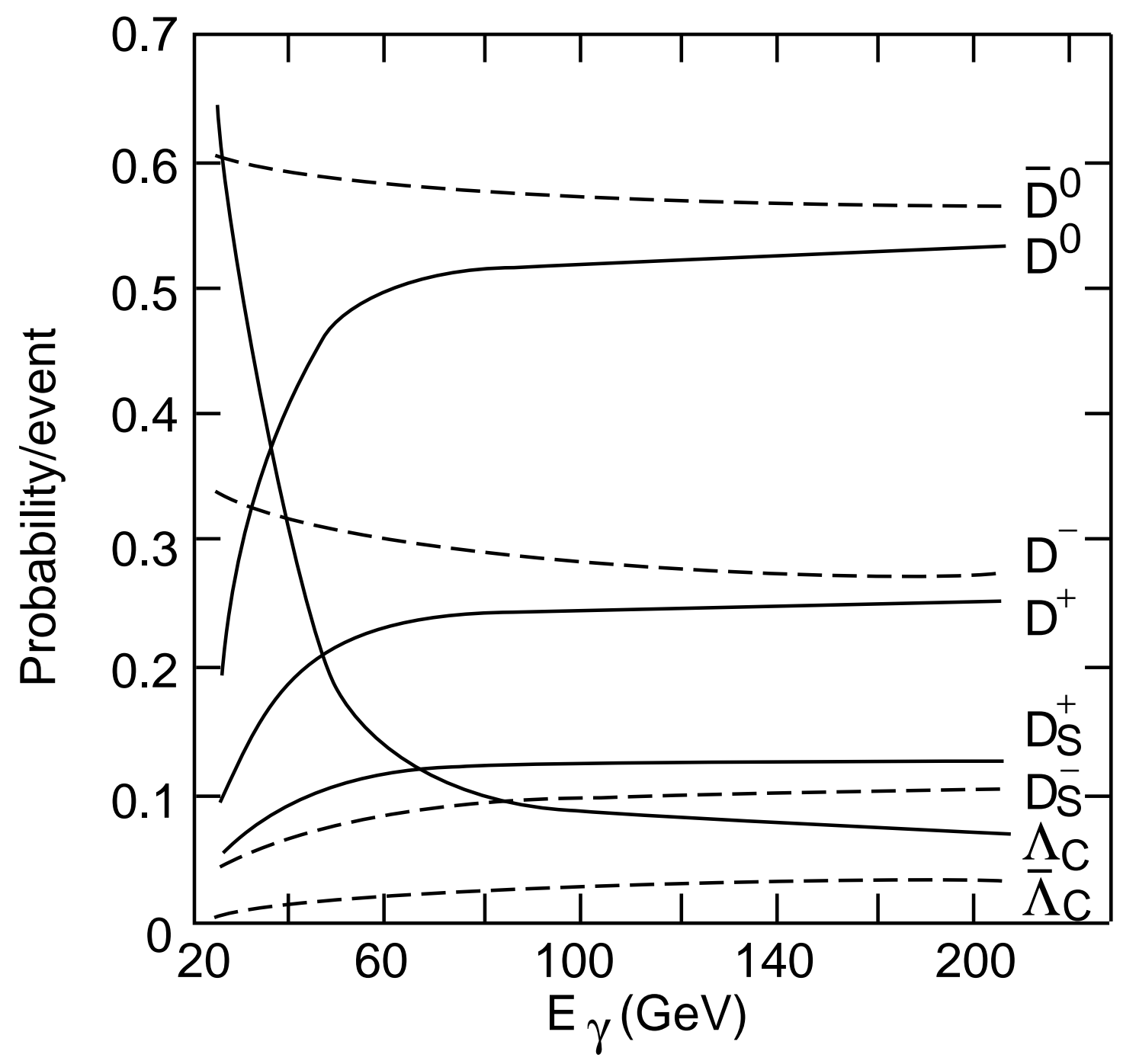

Figure 3 


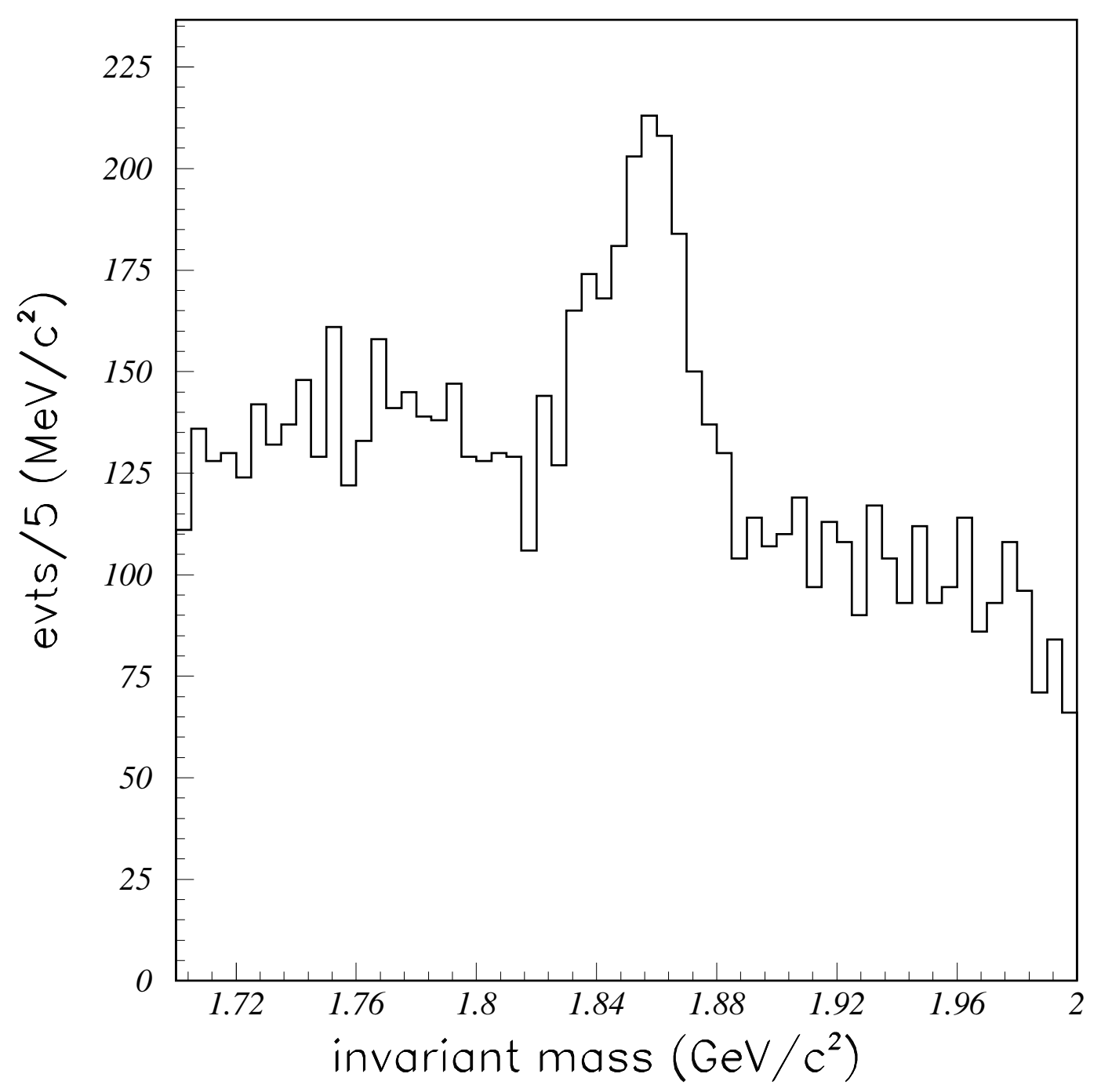

Figure 4 


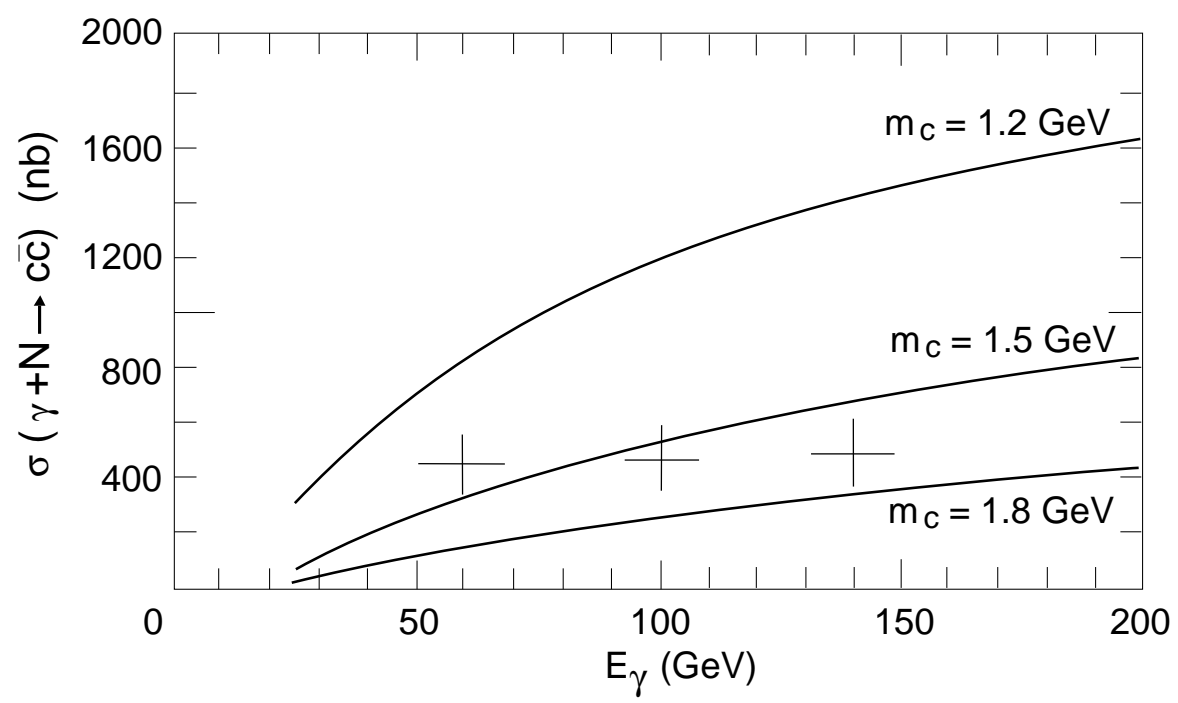

Figure 5
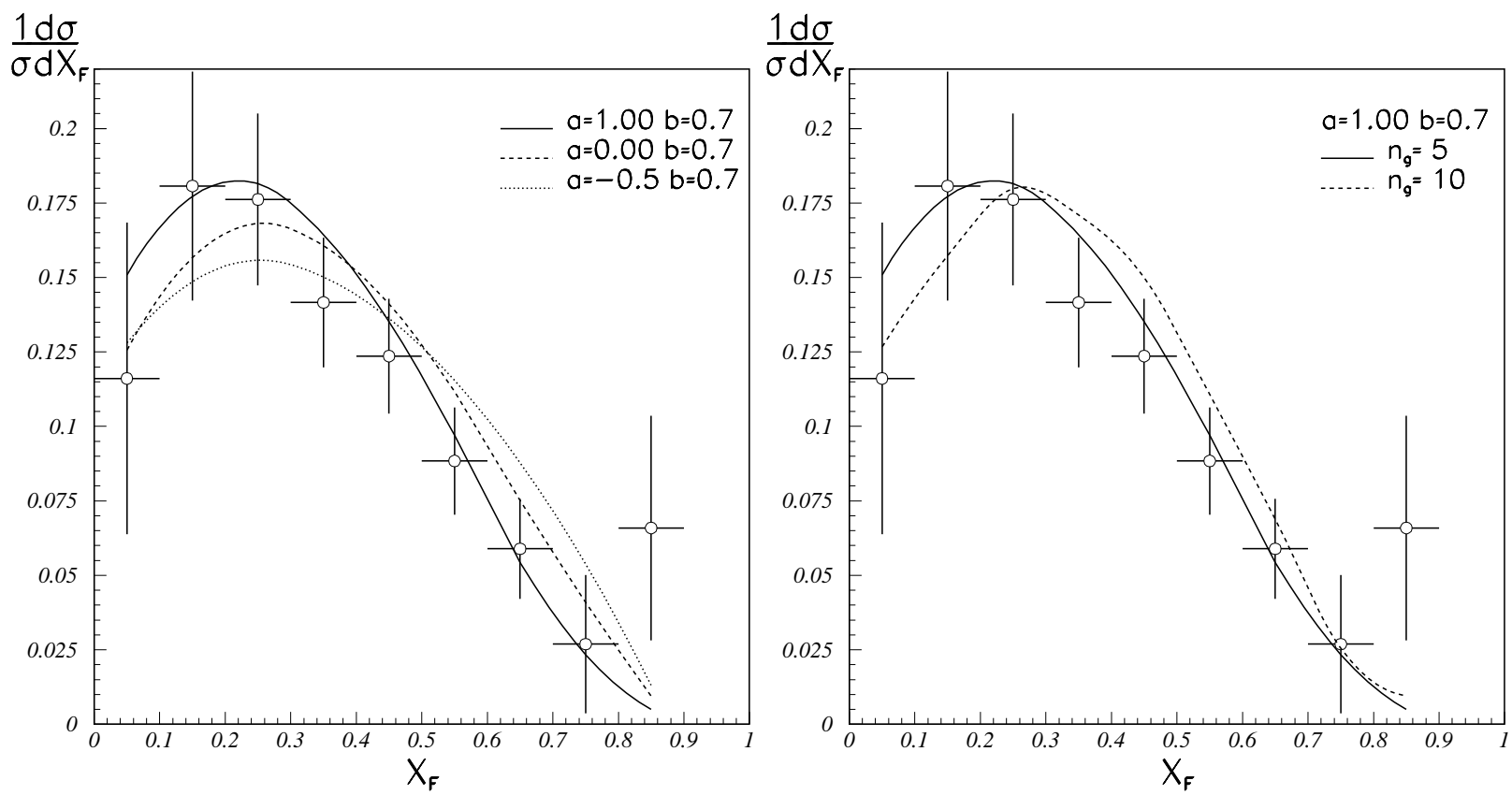

a

Figure 6 


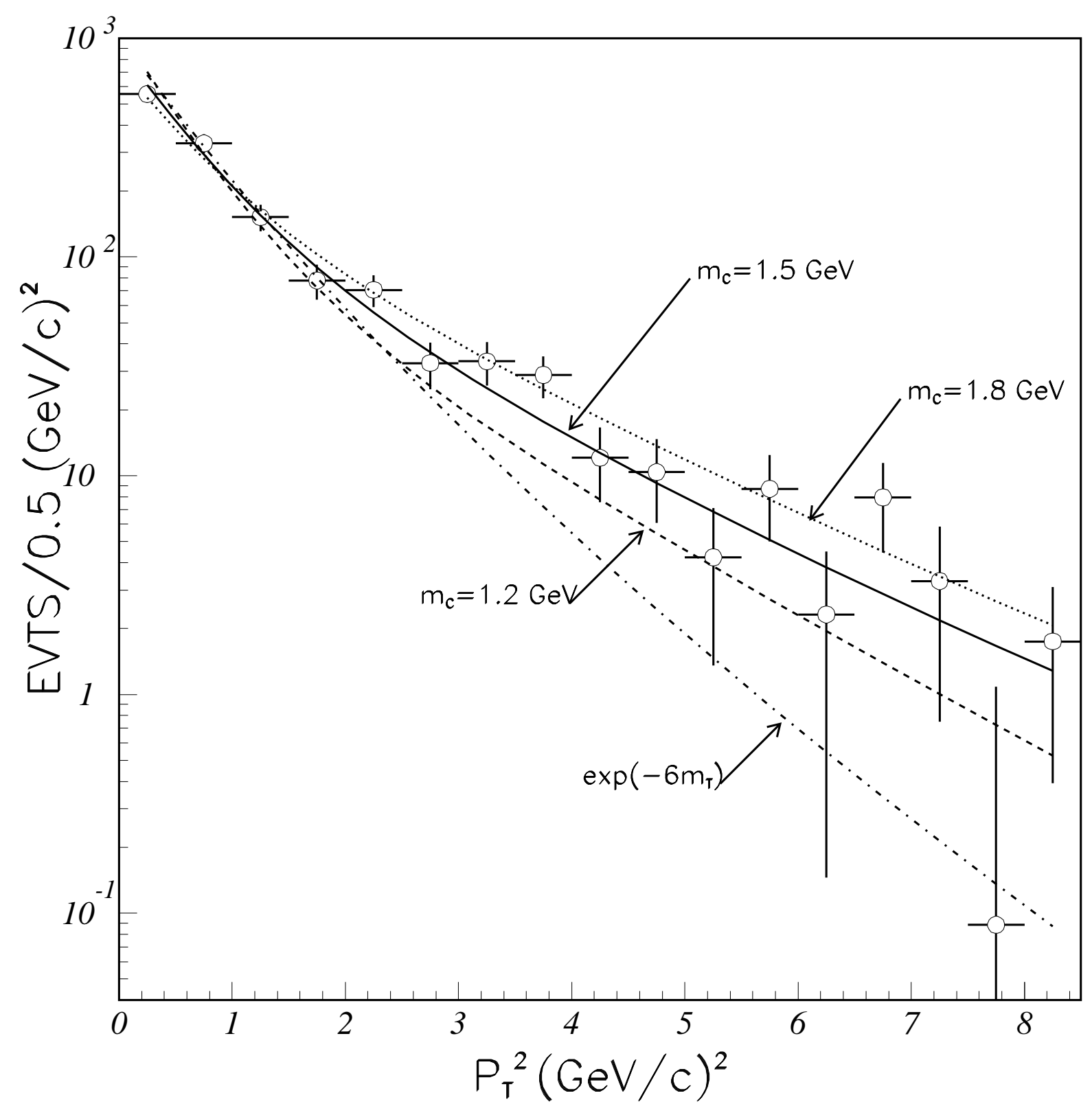

Figure 7 